\title{
Field-Trial of an All-Optical PSK Regenerator/Multicaster in a 40 Gbit/s, 38 Channel DWDM Transmission Experiment
}

\author{
R.Slavík $^{1,2}$, A.Bogris ${ }^{3,4}$, J.Kakande ${ }^{1}$, F.Parmigiani ${ }^{1}$, L.Grüner-Nielsen ${ }^{5}$, R.Phelan ${ }^{6}$, .Vojtech $^{7}$, \\ P.Petropoulos ${ }^{1}$, D.Syvridis ${ }^{3}$, D.J.Richardson ${ }^{1}$ \\ 1. Optoelectronics Research Centre, University of Southampton, UK \\ 2. on leave from the Institute of Photonics and Electronics, v.v.i., AS CR, Prague, Chaberska 57, \\ Czech Republic \\ 3. Department of Informatics and Telecommunications, National and Kapodistrian University of \\ Athens, Panepistimiopolis, llisia, Athens, GR-15784, Greece \\ 4. Department of Informatics, Technological Educational Institute of Athens, Aghiou Spiridonos, \\ Egaleo, 12210, Greece. \\ 5. OFS Priorparken 680, 2605 Brondby, Denmark. \\ 6. Eblana Photonics Inc., Dublin, Ireland. \\ 7. CESNET a.l.e., Prague, Czech Republic
}

\begin{abstract}
The performance of future ultra-long haul communication systems exploiting phase-encoded signals is likely to be compromised by noise generated during signal transmission. One potential way to mitigate such noise is to use Phase Sensitive Amplifiers (PSAs) which have been demonstrated to help remove phase as well as amplitude noise from phaseencoded signals. Recently, we showed that a PSA-based signal regenerator based on degenerate four-wave mixing can be implemented in a network-compatible manner in which only the (noisy) signal is present at the device input (black-box operation). The developed regenerator was also able to perform simultaneous wavelength conversion and multicasting, details/analysis of which are presented herein. However, this scheme was tested only with artificial noise generated in the laboratory and with the regenerator placed in front of the receiver, rather than in-line where even greater performance benefits are to be expected. Here, we address both theoretically and experimentally the important issue of how such a regenerator, operating for convenience in a multicasting mode, performs as an in-line device in an installed transmission fiber link. We also investigate the dispersion tolerance of the approach.
\end{abstract}

Index Terms- Optical signal processing, Fiber optics, Four-wave mixing, Optical amplifiers, Phase shift keying

This research has received funding from the European Communities Seventh Framework Programme FP/2007-2013 under grant agreement 224547 (PHASORS) and 255368 (TOP CLASS). We acknowledge the support of JISC and JANET (UK) for the funding and provision of the JANET-Aurora dark-fiber facility. Dr. F. Parmigiani gratefully acknowledges the support from the Royal Academy of Engineering/EPSRC through a University Research Fellowship. 


\section{INTRODUCTION}

$\mathrm{M}$ ODERN longhaul optical communication systems exploit both the amplitude and phase of the optical carrier to achieve higher spectral efficiencies and thus higher overall data capacities [1],[2]. Encoding the signal in phase also brings better resilience to certain linear and nonlinear impairments [3]. However, phase noise introduced during transmission both from optical amplifiers and the nonlinear interactions between channels can represent a limiting factor to data transmission [4]. Phase sensitive amplification (PSA) has been widely recognized as an effective way to regenerate phase-encoded signals, thanks both to its phase squeezing capabilities [5], [6], [7] and the ability to regenerate the signal amplitude when operated in the saturated regime [5].

In PSA, the phase relationship between the required pump(s), signal and any idlers present needs to be maintained, which is challenging in practice due to the carrier-less nature of phase encoded signals. Moreover, even if the carrier could be extracted it would generally contain a component of the phase and amplitude noise of the transmitted data. Recently, we demonstrated schemes [8], [9], [10] in which the carrier from a carrier-less PSK signal was recovered with minimal transfer of noise from the signal [11],[12] and then used to locally generate pump beams phase locked to the incoming data prior to signal regeneration in a degenerate two-pump PSA [7]. The regenerator was also able to perform simultaneous regeneration and multicasting/wavelength conversion [10]. However, so far, the device was tested only with artificial noise generated in the laboratory and with the regenerator placed immediately in front of the receiver, whereas its natural position would be in-line. This is because an in-line regenerator can reduce the build up of amplitude and phase noise directly in the optical domain allowing for all-optical extension of the fiber transmission reach.

Here, we address both theoretically and experimentally several key features of the developed regenerator. Some of the results were presented in less detail in [13]. Specifically, (i) how the regenerator works as an in-line device, (ii) how it works in an installed transmission line subject to real world noise, (iii) its multicasting and wavelength conversion capabilities, and finally, (iv) its tolerance to residual (uncompensated) link dispersion.

The paper is structured as follows. In Section II we briefly review some of the basic principles underpinning our field experiments on DPSK regeneration, (specifically phase sensitive amplification and multicasting/wavelength conversion), and also provide a brief summary of the theoretical approaches used to model our experiments. In Section III we describe theoretical and experimental work on regenerative multicasting which shows that this technique is appropriate for use in our studies. In Section IV we describe the theoretical basis and expectations for our field experiments and describe the transmission line configuration and regenerator used. We then describe the experimental results obtained that quantify the performance benefits obtained using the regenerator used both at the receiver and as an in line device. In Section $\mathrm{V}$ we draw our conclusions.

\section{PRINCIPLES}

\section{A. Phase Sensitive Amplification}

In PSA, the two quadrature components of the electric field of the signal experience different amounts of gain. An example is the phase-sensitive degenerate four-wave mixing (FWM)-based parametric amplifier, Fig. 1 , where the in-phase field component experiences gain $g$, while the quadrature component is de-amplified by $1 / g$. Consequently, the output phase of the amplified signal is more closely aligned towards the amplifier's in-phase axis [7], [9]. This effect, known as 'phase squeezing', is inherently suitable for the regeneration of binary phase-encoded signals, as the 'phase squeezed' data bits are forced to adopt a phase of 0 or $\pi$, in accordance with their phase prior to transmission thereby restoring the fidelity of the original data signal [5]. By operating the PSA in the saturation regime one can obtain simultaneous phase and amplitude regeneration, although this slightly compromises the degree of phase squeezing.

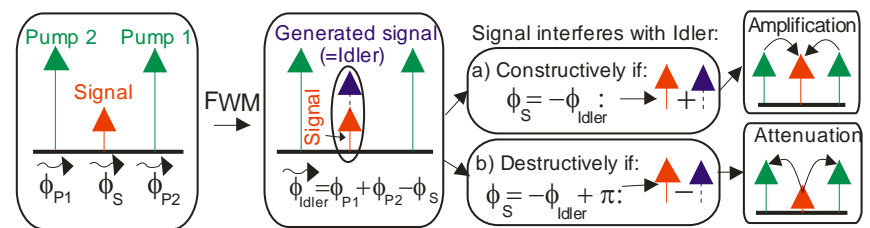

Fig. 1 PSA based on a degenerate four wave mixing. The generated idler is at the same wavelength as the signal. These two can interfere constructively or destructively, depending on the phase of the signal. It maximally amplifies the signal when the phase of the signal is either 0 or $\pi$ relative to the phase of the pumps (in-phase components), and maximally attenuates the signal when this phase is either $\pi / 2$ or $3 \pi / 2$. (quadrature components).

\section{B. Multicasting/wavelength conversion}

Wavelength multicasting, where a signal is simultaneously replicated at several wavelengths, is potentially a useful technique for future networks, combining essential functionalities for WDM systems, such as wavelength routing and monitoring, with broadcasting (as required, for example, for the distribution of high-definition video to multiple networks). Parametric processes have already been shown to be suitable this purpose with multicasting of up to 40 channels already demonstrated [14]. The degenerate PSA shown in Fig. 1 is also capable of providing multiple copies of the input signal at different wavelengths together with optical regeneration [10] when operated in the saturation regime. As sketched in Fig. 2, several FWM interactions may 
occur simultaneously: PSA, which involves the two pumps and the data, is responsible for the regeneration (Fig. 2a), whereas new (secondary) pumps as well as new copies of the data signal are generated via phase insensitive FWM interactions (Fig. 2b). In the saturation regime, the high input signal power will give rise to the generation of multiple FWM products which will inherit the amplitude and phase properties of the initially interacting waves (Pump 1, Pump 2, and Data). Specific products such as Copy -1 and Copy 1 and their higher order harmonics are either exact or conjugate replicas of the signal and thus carry the input data. Hence, the regenerative performance of the PSA which is imprinted at the signal wavelength is also expected to be transferred to these specific FWM products. Analysis supporting this description is given in Section III.
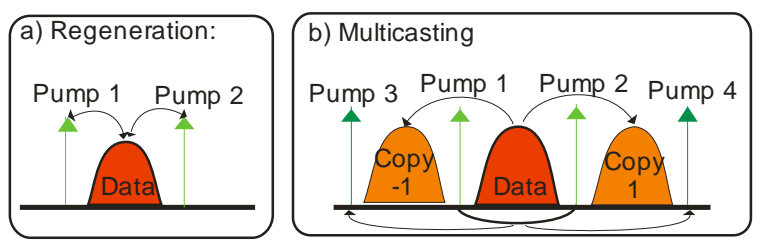

Fig. 2 In a $\chi^{3}$ non-linear medium, several FWM processes are present such as (a) PSA and (b) generation of secondary pump and signal copies via phase insensitive FWM.

\section{Theoretical analysis}

The regenerator considered in our theoretical analysis and experimental studies is based on the degenerate two-pump PSA system, which has been described in detail in [9]. It consists of two parametric amplifiers with a noise removal stage in between. The first parametric amplifier operates in a phase-insensitive mode generating a modulation-stripped idler phase-locked to the pump and data signal. The noise removing stage consists of an injection locked laser that serves to amplify the idler and to remove any amplitude noise and high-frequency phase noise associated with it [15] to obtain phase locked, cw pumps for the final stage PSA where the phase and amplitude regeneration of the incident data signal occurs. The nonlinear interaction between the signal and the pumps within these two highly non-linear fiber (HNLF) based parametric amplifiers was numerically simulated with the use of the Split-Step Fourier method for the numerical integration of the nonlinear Schrödinger equation [16]. The lasers and injection locking were simulated based on a rate equation model [17] in order to take into account their noise and coherence properties. The parameters used in the numerical simulations are similar to those of the real devices (HNLFs, lasers) described in the experimental part of Section IV. The simulation results shown here have a complementary role to the experimental study. They were carried out to show how the regenerator would handle impairments arising from strong accumulated nonlinearities, as would be observed had our link been longer or operated outside of the optimal regime.

\section{Multicasting}

For practical reasons that will become clear in Section IV the use of multicasting within our in-line field experiments on the regenerative properties of our black-box regenerator was essential. We therefore first conducted simulations and experiments to establish how the basic regenerative properties of our device applied both to the incoming signal and higher order (wavelength shifted) multicasting components.

\section{A. Theoretical analysis}

The noise of the input signal to our multicasting regenerator (see Fig.5 for configuration) was emulated by numerically propagating an initially pristine $40 \mathrm{Gbit} / \mathrm{s}$ DPSK signal with average power of $0 \mathrm{dBm}$ through a 10 -channel, $100-\mathrm{GHz}$ spaced 400 $\mathrm{km}$ long fiber link. To obtain significant amplitude and phase noise levels, the average power at the input of the link was $0 \mathrm{dBm}$ sufficiently high to see the emergence of nonlinear phase noise. The PSA operated in the saturation regime with the input signal power being equal to a quarter of the combined pump power. The total power at the input of the $177 \mathrm{~m}$ long HNLF was equal to $31 \mathrm{dBm}$ (see Section IV for more details on other HNLF parameters).

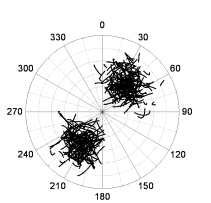

a)

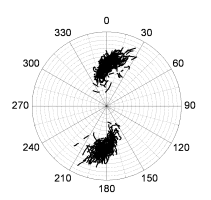

b)

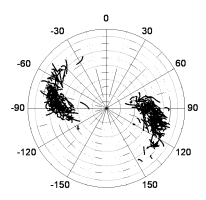

c)

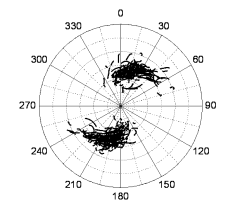

d)

Fig. 3 Constellation diagrams of a) the input signal, b) output signal (Copy 0), c) output Copy -1 and d) output Copy -2.

According to the results shown in Fig. 3, regeneration of both the phase and amplitude was observed for the signal (Copy 0). The numerical analysis showed that the input phase standard deviation of 50 deg was reduced to 19, 25, and 36 deg for Copy 0 , -1 and -2 , respectively. The more limited reduction in the phase noise of the generated copies is attributed to the fact that they are formed by a combination of phase sensitive and phase insensitive processes. In support of this hypothesis, Fig. 4 presents 
constellations at the middle of the HNLF. Within the first half of the HNLF $(88.5 \mathrm{~m})$, Copies 0 and -1 have mostly benefited from the phase sensitive interaction whilst Copy -2 still resembles the input signal of Fig. 3a. Another explanation for the degradation of the higher order copies relates to the intrinsic lower signal-to-noise ratio (SNR) of the higher order FWM copies and their dependence on the similarly degraded higher order pumps.

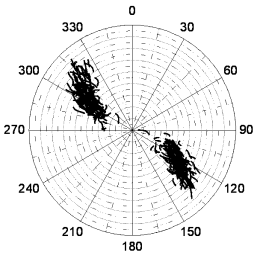

a)

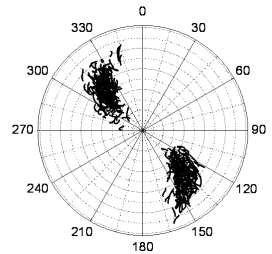

b)

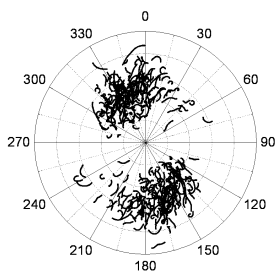

c)

Fig. 4 Constellation diagrams of a) the output signal, b) output Copy -1 and c) output Copy -2 at the first 88.5m of the 177m long HNLF.

\section{B. Experimental Set-up}

The regenerative multicaster set-up used is almost identical to that presented in [8] and is shown in Fig. 5. The only difference is the demultiplexer at the output that now allows separation and access to not only the original signal (Copy 0), but also the generated copies at different wavelengths. The data signal is a $40 \mathrm{Gbit} / \mathrm{s}$ non-return-to-zero (NRZ)-DPSK, $2^{31}-1$ pseudo-random bit sequence (PRBS). To study the phase regenerative capabilities, the signal phase was modulated in a deterministic fashion using an additional phase modulator, driven at a single-frequency close to $20 \mathrm{GHz}$.

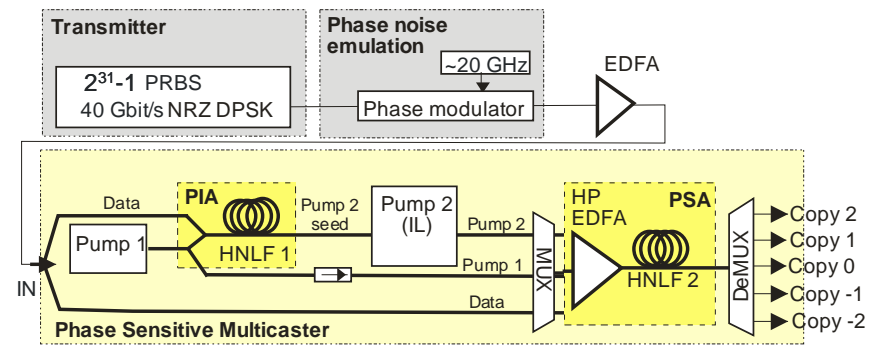

Fig. 5 Set-up for regenerative multicaster based on PSA._Pump 1: free running CW laser, Pump 2: injection-locked semiconductor laser.

Fig. 6 shows the PSA output spectrum for the optimal total power at the input of the HNLF of $31 \mathrm{dBm}$ (this is $\sim 3 \mathrm{~dB}$ higher than required for same-wavelength regeneration [9]). It consists of several copies of both the pumps (generated via FWM from the original input Pumps 1 and 2) and the signal. Here, we consider five copies, which have similar power levels: Copy 0 (at the original wavelength), Copies \pm 1 with about the same power as Copy 0 , and Copies \pm 2 with power about 2 dB less than Copy 0 .

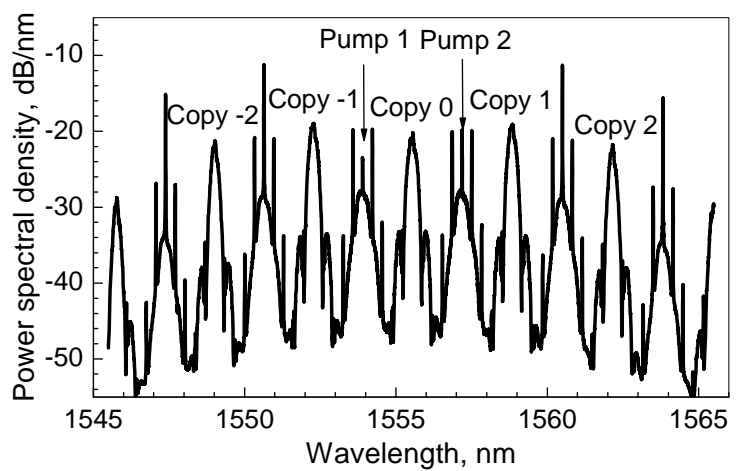

Fig. 6 Output PSA spectral characteristics. Signal at the input signal wavelength (Copy 0) and four copies with power difference of less than 2 dB are considered. 


\section{Experimental Results}

For the sake of simplicity, we present results for Copies 0 , -1, and -2, only. It is expected (and confirmed in our earlier modelling) that results for Copies 1 and 2 would be similar to those for Copies -1 and -2, respectively. We evaluated the performance of the system by measuring the bit error ratios (BERs) and eye diagrams.

First, we used a clean (no noise) input data signal. The eye diagrams are shown in Table 1 (upper part) and the BER curves in Fig. 7a. As also observed previously [8], Copy 0 has a slightly negative power penalty as compared to the back-to-back (about 1 $\mathrm{dB}$ ). Analysis of the corresponding eye diagrams in Table 1 suggest that the shape of the demodulated pulse is different at the PSA input/output, which may explain this slight improvement in the receiver power sensitivity. BER measurements of the other copies show error free operation with power penalties less than $1 \mathrm{~dB}$ as compared to the back-to-back (and less than $3 \mathrm{~dB}$ as compared to Copy 0).

Table 1: Eye diagrams of Copy 0,-1 and -2 for noise off (left) and on (right), respectively.

\begin{tabular}{|c|c|c|c|c|}
\hline Noise & Input & Copy 0 & Copy -1 & Copy -2 \\
\hline OFF & & & & \\
& & & & \\
\hline ON & & & & \\
\hline
\end{tabular}

Subsequently, we introduced phase distortion on the data at the input of the multicasting scheme, severely degrading the corresponding BER curve and eye diagram at the input of the multicasting scheme (Fig. 7b and Table 1) - a power penalty of 13 $\mathrm{dB}$ was obtained at the BER $10^{-7}$ level (Fig. 7a and b). The BER curves for all of the characterized copies (Fig. 7b) exhibit better receiver sensitivity as compared to the input signal clearly demonstrating the regenerative property of the presented scheme. This regenerative performance is also visible from the corresponding eye diagrams shown in the second row of Tab. 1 .
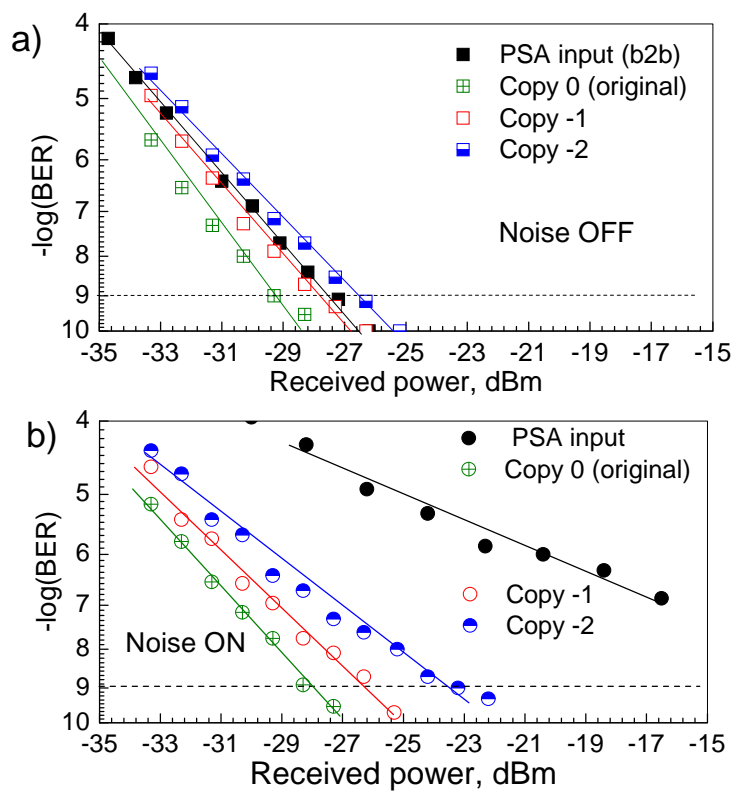

Fig. 7 BER curves of Copy 0,-1 and -2 for noise off (a) and on (b), respectively.

Our experiments thus confirm the theoretical prediction that the regenerative properties of the regenerator that apply to the original signal extend to the wavelength shifted multicasted copies with just a relatively minor reduction in performance that increases with order number. 


\section{TRANSMISSION EXPERIMENT}

\section{A. Theoretical analysis}

In this Section we first concern ourselves with the regeneration experienced at the original signal wavelength (Copy 0) with a regenerator placed right in the middle of the transmission link, Fig. 8. The regenerator operates on one 40-Gbit/s DPSK channel in a DWDM system with $100 \mathrm{GHz}$ channel spacing. For simplicity, propagation of just 10 channels was considered, with the regenerated channel being in the middle of the spectral allocation as it is expected that the middle channels will be impaired the most by cross phase modulation (XPM) effects. The regenerator was optimized (pumps and input data signal powers) to obtain simultaneous regeneration in both phase and amplitude [9], [18], as will be discussed in detail later in this Section.

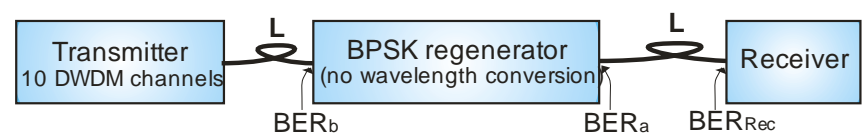

Fig. 8 Conceptual scheme of the in-line regeneration of DPSK signals with the use of PSAs. BER $\mathrm{b}_{\mathrm{b}}, \mathrm{B}_{\mathrm{a}}$ and $B E R_{\mathrm{Rec}}$ stands for the BER value before and after PSA, and at the receiver, respectively.

The numerical simulations calculated the BER through an error counting approach. This technique is very reliable as it does not rely on estimations of the probability density function of the superposition of all noise sources (linear, nonlinear, etc) that impair the signal. Its main drawback however is that it is time consuming, so error counting down to the $10^{-9}$ level is computationally intensive. Error counting was carried out through 1000 repetitions of the transmission of 10000 bits of ten independent DPSK signals with a $100 \mathrm{GHz}$ wavelength spacing. Error counting calculates the BER floor of the transmission system ignoring the impact of the noise and any deficiencies of the balanced differential receiver.

We consider the most important criterion for the evaluation of a regenerator to be the extent to which the transmission reach is increased. Thus, we compare the BER in front of the regenerator $\left(B E R_{b}\right)$ with that at the receiver of an extended link of length $2 L$ $\left(B E R_{\text {Rec }}\right)$, Fig. 8. In this case, doubling of the transmission reach requires that $B E R_{b}$ is equal to $B E R_{\text {Rec }}$. Additionally, $B E R_{a}$ should be lower than $\mathrm{BER}_{\mathrm{b}}$, otherwise the regenerator would not offer true restoration of the input signal. For all other optical regenerators which cannot improve the BER performance, the best anticipated performance for $B_{E R} R_{\text {ec }}$ is two times $B E R_{b}$ [19]. The BER performance of both the regenerated and the non-regenerated link along the line is compared in Fig. 9, considering two different channel power levels and the regenerator placed at $L=400 \mathrm{~km}$, in line with the experimental study. The figure shows that the BER performance deteriorates with increasing power per channel, which indicates that the transmission system is mainly impaired by nonlinear noise. In both cases, the regenerator improves the BER by at least one order of magnitude. As mentioned above, the most interesting comparison is between $B R_{b}$ and $B E R_{R e c}$ which evaluates the restoration potential of the regenerator. $\mathrm{BER}_{\mathrm{b}}$ is only slightly lower than $\mathrm{BER}_{\mathrm{Rec}}$ which means that the regenerator is capable of almost doubling the reach of the transmission system. The main reasons for the slightly worse performance of the second span in comparison to the first are the accumulated noise affecting the neighboring channels and the amplitude regeneration properties of the PSA. As the other channels are not regenerated in the mid-span, the contribution of noise attributed to XPM is expected to be higher within the second span.

It is worth noting that the power levels considered here are higher than those used in the experiment; if similar values were used, BER floor levels at $<10^{-7}$ would be obtained, but as pointed out above, this would require unrealistically long computation times. Therefore the results presented here should be used as a guide only for the performance anticipated in the experiment. It is also useful to note that the regenerator is expected to work better at higher levels of transmitted power, or in longer fiber links, where more non-linear noise is present [9].

We next analyze the PSA performance limitations when operated to provide simultaneous amplitude and phase regeneration [8], [9], [20]. As we show below, the limitations arise from the fact that the amplitude regeneration requires PSA saturation which unfortunately reduces the PSA phase regeneration capability. Fig. 10 shows the constellation diagrams of the signal before (a) and after the PSA (b,c,d) for different input signal power levels, which influences the level of saturation (the higher the input power with respect to the powers of the two pumps, the higher the saturation). 

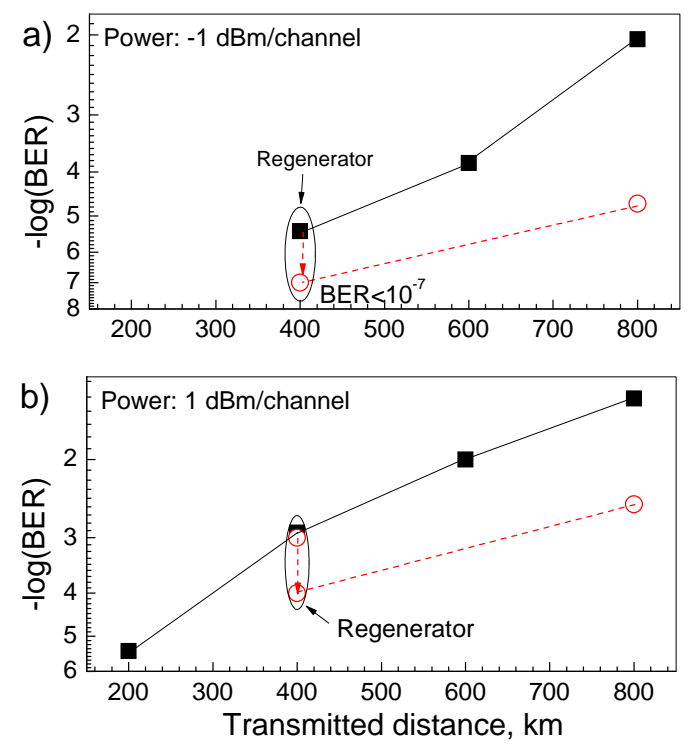

Fig. 9 BER performance of WDM transmission links with (open circles) and without (black squares) in-line mid-span regenerator for two average power-per channel: a) $-1 \mathrm{dBm}$, and b) $1 \mathrm{dBm}$.

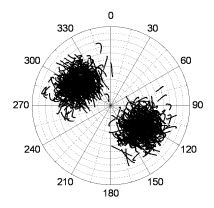

a)

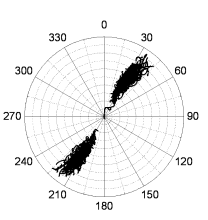

b)

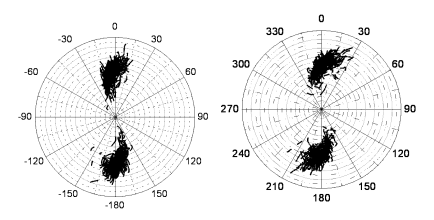

d)

Fig. 10 Constellation diagrams of (a) the signal at $400 \mathrm{~km}$ and the output of the PSA regenerator for input signal power equal to (b) $60 \mathrm{~mW}$, (c) $240 \mathrm{~mW}$ and (d) $360 \mathrm{~mW}$. The transmitted power per channel is $0 \mathrm{dBm}$.

Although optical dispersion compensation is currently applied to long-haul transmission links, perfect cancellation of the accumulated dispersion for the entire spectral transmission window including higher-order dispersion is not straightforward. For this reason, we conducted a numerical analysis to quantify the BER performance of a PSA-based DPSK regenerator for different levels of residual dispersion. Similar to the previous study, the signal noise was obtained via propagation through $400 \mathrm{~km}$ DWDM system.

(a)

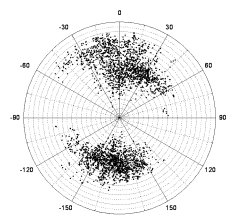

(b)

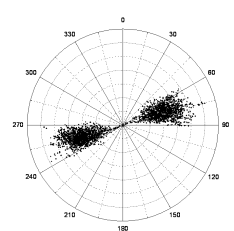

Fig. 11 Constellation diagrams of a $40 \mathrm{~Gb} / \mathrm{s}$ DPSK signal (a) before and (b) after the regenerator. The signal has experienced residual dispersion of 80 ps/nm.

The figure clearly shows that the PSA is capable of reducing the phase noise fluctuations at the expense of amplitude stability. The amplitude can even reach the zero level depending on the input signal phase. The large amplitude fluctuations affect the BER performance of the PSA as depicted in Fig. 12, where the BER is calculated as a function of the uncompensated distance in SMF-28 for two different levels of signal noise (corresponding to $1 \mathrm{dBm}$ and $2 \mathrm{dBm}$ of launched power into the link, respectively). We see that the PSA can tolerate an accumulated dispersion of $100 \mathrm{ps} / \mathrm{nm}$ at $40 \mathrm{~Gb} / \mathrm{s}$ whilst offering better BER performance compared with the non-regenerated dispersion impaired signal. 

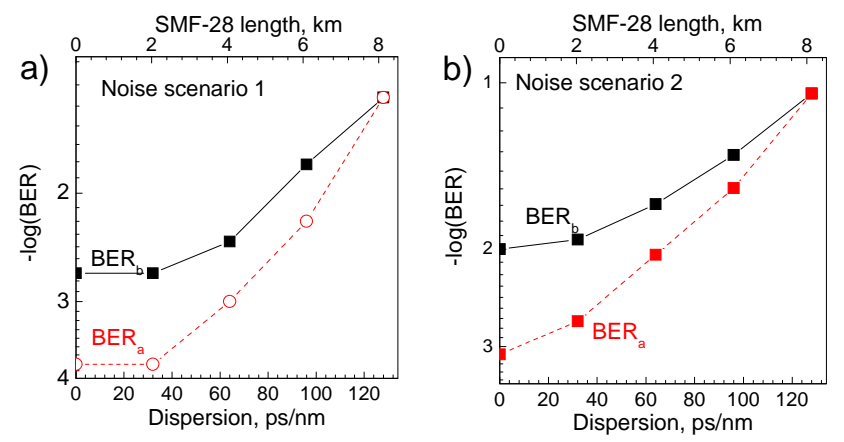

Fig. 12 BER performance of a dispersion limited PSA regenerator for two noise scenarios in front of a DPSK receiver. BER $\mathrm{a}_{\mathrm{a}, \mathrm{b}}$ refers to 'before' and 'after' the regenerator, respectively.

\section{B. Experimental validation}

We next present our field transmission experiments, which have made use of the wavelength conversion/multicasting feature of the regenerator. A conceptual outline of our network and its practical implementation are shown in Fig. 13a and Fig. 13b respectively. At the transmitter, we combined $37 \mathrm{CW}$ semiconductor lasers on a $100 \mathrm{GHz}$ DWDM ITU grid and modulated them with $40 \mathrm{GHz}, 2^{31}-1$ PRBS DPSK data. To de-correlate adjacent channels, we split the odd/even channels in an interleaver, introduced $55 \mathrm{~ns}$ of relative delay, and then re-combined them. In order to facilitate placing the regenerator at the mid-point of our network and to operate at twice the transmission distance that was physically available to us, we chose to incorporate a wavelength shift in the regeneration process, Fig. 13b. Obviously, conversion of the wavelength would not normally be required in most transmission applications, nevertheless, it serves to illustrate another useful functionality of the technology (wavelength conversion/multicasting) as previously reviewed [10]. 37 channels (excluding ITU Channel 23) were sent down the dark fiber link (part of the UK JANET Aurora Network) that extends from Southampton to London and back again (400 km dispersioncompensated transmission distance, 6 in-line flat-gain EDFAs with maximum input/output powers of $-5 / 15 \mathrm{dBm}$, operated in automatic gain control, nominal gain $20 \mathrm{~dB}$ ). The maximum total power launched into the link was $7 \mathrm{dBm}$ with maximum power along the link of $15 \mathrm{dBm}$. The detailed configuration is shown in Fig. 14. At its output, we dropped ITU Channel 27, performed wavelength conversion to the slot of Channel 23 [Copy -1] (either with or without regeneration) and sent it through the link again with all other channels. Therefore a total of 38 channels occupied the frequency grid at any time.

a)

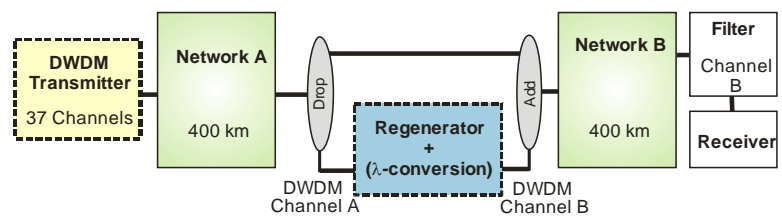

b)

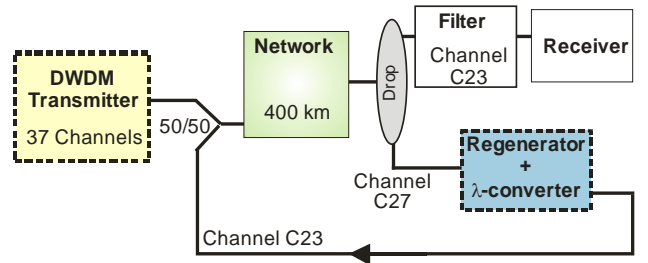

Fig. 13 Model experiment with regenerator as an in-line device (a) and the implemented network that emulates key features of the model network (b).

A basic schematic of our regenerator is shown in Fig. 15 and is almost identical to that used in [9]. HNLF1, used in the phase insensitive stage, was $500 \mathrm{~m}$ long with a dispersion of $0.09 \mathrm{ps} / \mathrm{nm} / \mathrm{km}$, nonlinear coefficient of $11.5 \mathrm{~W}^{-1} \mathrm{~km}^{-1}$, and an attenuation of $0.8 \mathrm{~dB} / \mathrm{km}$. HNLF2, which was used in the PSA was an alumino-silicate fiber with an applied strain gradient to increase the Stimulated Brillouin Scattering (SBS) threshold to $>28 \mathrm{dBm}$ thereby avoiding the need for any active SBS suppression scheme. This fiber was $177 \mathrm{~m}$ long with a dispersion of $0.13 \mathrm{ps} / \mathrm{nm} / \mathrm{km}$, nonlinear coefficient of $7.1 \mathrm{~W}^{-1} \mathrm{~km}^{-1}$, and an attenuation of $15 \mathrm{~dB} / \mathrm{km}$. The very low dispersion and dispersion slope values facilitate the generation of multiple copies of the signal and pumps. The total power at the input of HNLF 2 was $31 \mathrm{dBm}$. The injection locked laser was a discrete-mode semiconductor laser from Eblana Inc., Ireland with a natural linewidth of $300 \mathrm{kHz}$ [24]. Pump 1 was a laser with natural linewidth below $10 \mathrm{kHz}$. 


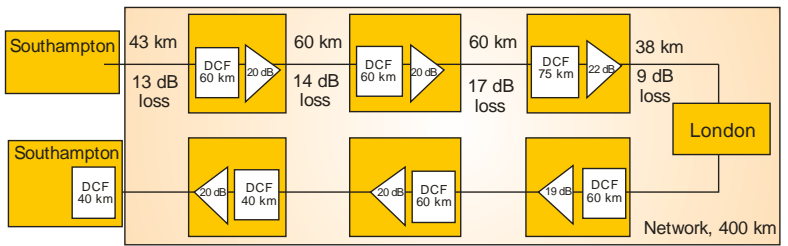

Fig. 14 Dark fiber link configuration. It consists of two times four spans (roundtrip) - lengths and attenuations ares shown in the figure. Amplifiers are operated in automatic gain control - the gain value is shown. DCF: dispersion compensating fiber - the number shown refers to how many km of SMF-28 it compensates.

The regenerator was configured to perform simultaneous regeneration and multicasting and was mounted into a standard telecommunication rack along with other network components and test gear. To compensate for polarization drift in the network, an endless polarization tracker was incorporated into the system. To enable comparative study with/without regeneration, conventional wavelength conversion could be carried out by switching off Pump 2, resulting in phase insensitive FWM-based wavelength conversion.

The receiver consisted of a 0.6-nm bandpass filter, an attenuator (the received power was measured at its output), a low-noise EDFA, a bandpass filter, a 1-bit delay interferometer (DLI), and a balanced photodetector.

\section{Results}

We first transmitted the signal through $400 \mathrm{~km}$ of fiber (Fig. 13b without Channel C23 recirculating back) and compared the performance of the wavelength converted signal with (Pump 2 turned on) or without regeneration (Pump 2 turned off) to that obtained without transmission, Fig. 16. The regenerator fully restored data fidelity giving an improvement of $1.5 \mathrm{~dB}$ at the receiver for a $\mathrm{BER}=10^{-9}$. Slight reshaping of the eye is also observed, Fig. 16a, which may be responsible for the observed slight improvement of the BER curve as compared to that measured without transmission. Fig. 16b shows the improvement provided by the regenerator as we varied the total power launched into the link (for a fixed power at the receiver). For identical performance, around $2 \mathrm{~dB}$ less total power could be launched to the link when the regenerator was used. Since the BER is shown to improve monotonically with the launched power, we believe that the noise generated in the link was dominated by linear (ASE) rather than non-linear noise. This is a consequence of using a large number of channels (low power per-channel) and propagation over a relatively modest distance.

Results obtained after two round-trips through the link, Fig. 13b, (800 km, regenerator used "in-line") are shown in Fig. 17. Use of a mid-point regenerator was capable of reducing the BER power penalty by a factor of two (e.g., at $B E R=10^{-6}$, it reduces penalty from $2 \mathrm{~dB}$ to $1 \mathrm{~dB}$ and at $\mathrm{BER}=10^{-9}$, from $5 \mathrm{~dB}$ to $2 \mathrm{~dB}$ ). The error floor of the regenerated data is one order of magnitude lower than without regeneration, meaning that the regenerator prevents about $90 \%$ of errors in this regime. This is fully consistent with the earlier studies/theoretical analysis presented in [9], as well as with the theoretical analysis showed earlier. A closer study of the eye diagrams, Fig. 17a, shows about a 20\% larger eye opening for the regenerated signal. Varying the input power into the link, Fig. 17b, showed almost $4 \mathrm{~dB}$ power penalty in the second round trip. This value was reduced to 2 $\mathrm{dB}$ when the regenerator was used.

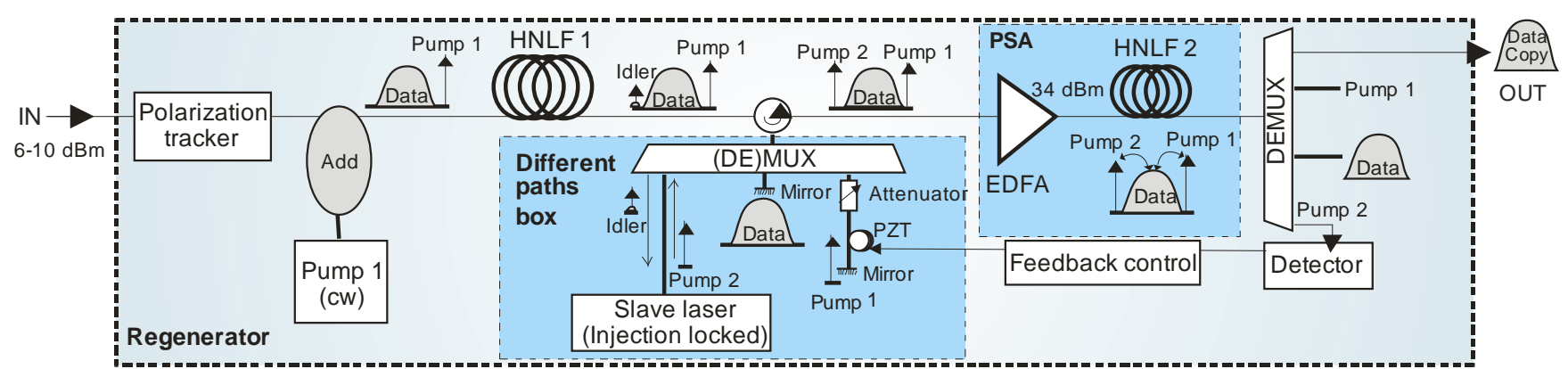

Fig. 15 Regenerator set-up. The data signal is mixed with a free-running cw laser (Pump 1) in HNLF 1, producing an idler phase-locked to the data/pump pair. The idler is amplified and regenerated via injection locking in the 'Different paths box', which is thermally/acoustically shielded to reduce acoustic/thermal pick-up experienced by signals propagating through separate fibers. Within this box, Pump 1 power is controlled via an attenuator and relative phase of the three waves (that may drift due to acoustic/thermal pick-up) is controlled via a PZT fiber stretcher. The pumps/data are subsequently amplified in an EDFA (erbium doped fiber amplifier) and sent to HNLF 2 for PSA. Pump 2 output signal is used for the PZT feedback keeping PSA at its operation point. 

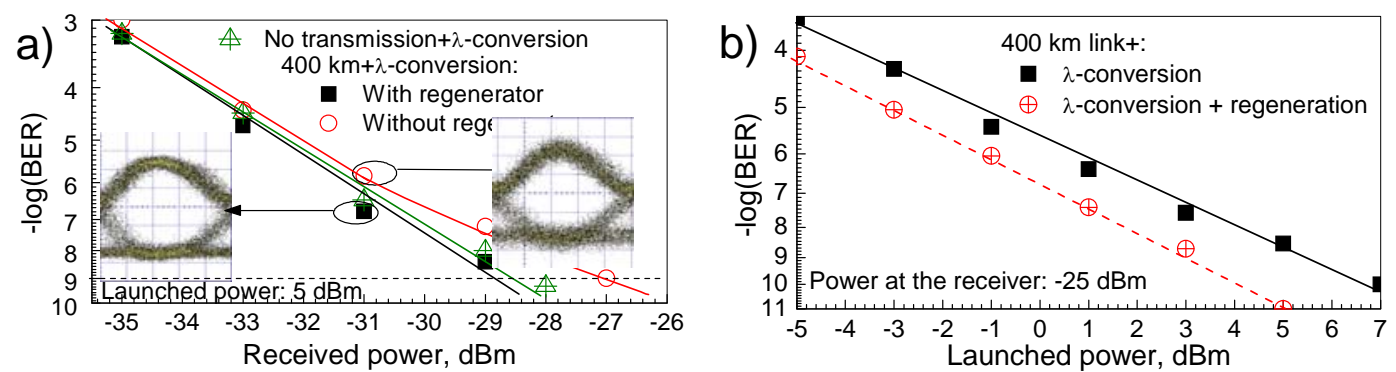

Fig. 16 BER curves at the output of the $1^{\text {st }}$ round-trip $(400 \mathrm{~km})$ measured at the data Channel 23. (a) Measured for the maximum power into the link $(7 \mathrm{dBm})$. For reference, a measurement of the $\lambda$-converted signal without transmission is also shown (green triangles). (b) BER measurement for various powers launched into the link and fixed receiver power of $-25 \mathrm{dBm}$.

Finally, we studied how the regenerator could cope with residual dispersion. We propagated a single 40 Gbit/s DPSK channel through one roundtrip $(400 \mathrm{~km})$ of fully dispersion-compensated dark fiber link, and subsequently added various lengths of SMF-28 fiber at its end. BERs were measured with and without the regenerator placed in front of the differential receiver, Fig. 18. For a fully compensated link, the sensitivity improvement (at $\mathrm{BER}=10^{-9}$ ) due to the regenerator was $2 \mathrm{~dB}$. The same result was obtained when $1 \mathrm{~km}$ of SMF-28 was added at the end of the link. However, for $2.5 \mathrm{~km}$ of SMF-28, a power penalty of $1 \mathrm{~dB}$ was obtained when using the regenerator, while without the regenerator the penalty rose to $4 \mathrm{~dB}$. When we tried to increase the SMF-28 length further to $8 \mathrm{~km}$, the use of the regenerator resulted in poorer performance as compared to the non-regenerated case. Thus, we can conclude that use of the regenerator can be beneficial for improving the system performance when small amounts of residual dispersion are likely to be present in the link. These results are in agreement with the numerical analysis given earlier in this Section, and are in line with previous theoretical studies of PSAs [25].
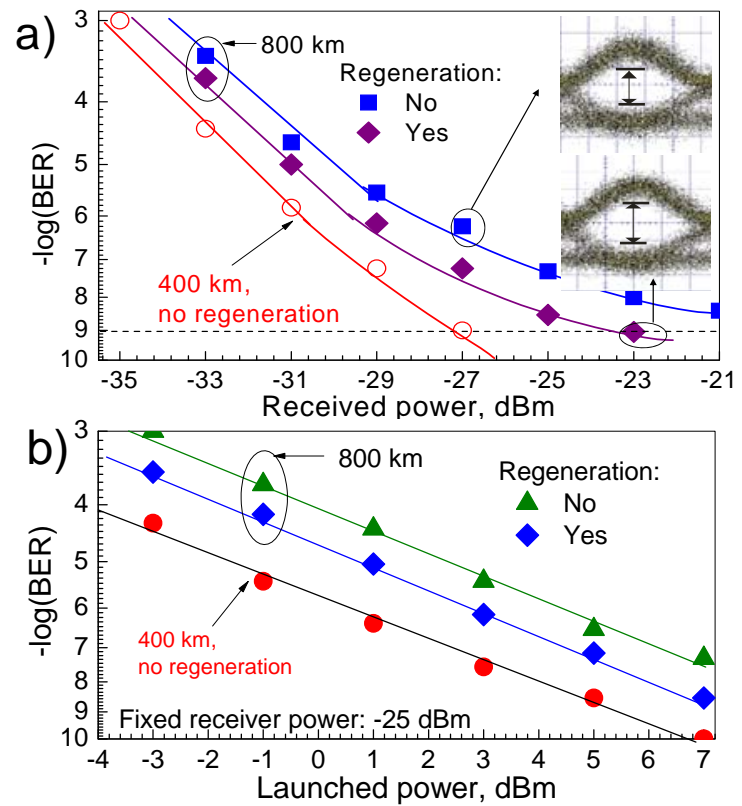

Fig. 17 BER curves at the output of the $2^{\text {nd }}$ round-trip $(800 \mathrm{~km}$ ) with and without mid-span regeneration: (a) for launched power of $5 \mathrm{dBm}$; (b) as a function of the launched power for fixed receiver power of $-25 \mathrm{dBm}$. For reference, measurements of the signal at the mid-point (after wavelength conversion) are also shown.

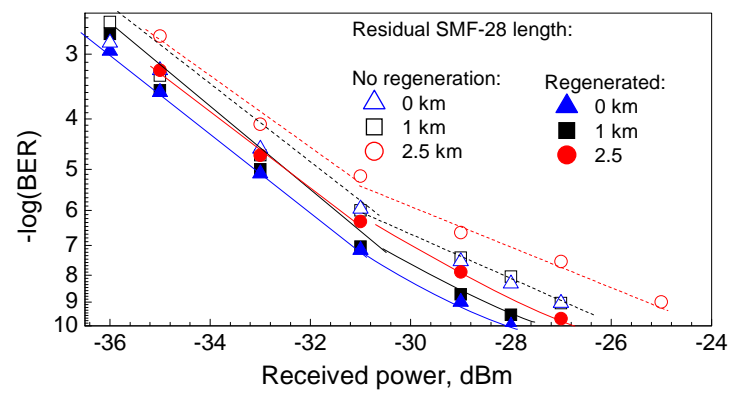

Fig. 18 BER curves at the output of the $1^{\text {st }}$ round-trip (400 km) using single-channel transmission when various length of dispersion-uncompensated SMF-28 fiber were added. 


\section{CONCLUSION}

We have studied several aspects of the performance of a recently-developed black-box regenerator for binary PSK signals both theoretically and experimentally. First, its capability to perform simultaneous regeneration and wavelength conversion was demonstrated. Theoretical analysis confirmed that this is possible through two cascaded FWM processes in the HNLF - first, the signal (Copy 0) is regenerated via PSA. Subsequently, new copies and secondary pumps are generated via phase insensitive FWM.

The regenerator was also tested as an in-line device in an installed transmission system that comprised 3840 Gbit/s DPSK channels. Due to experimental constrains, this was combined with the above-mentioned wavelength conversion function. Both theoretical analysis and experiments confirmed that inclusion of the regenerator allows extension of the reach of the regenerator by almost a factor of 2 . Indeed, the experiment showed that using the regenerator in the middle of the link allows $2 \mathrm{~dB}$ less power to be launched for identical BER performance.

The performance of the regenerator in the presence of residual chromatic dispersion was also tested and theoretically analyzed. For a $40 \mathrm{Gbit} / \mathrm{s}$ DPSK signal, the regenerator coped with $40 \mathrm{ps} / \mathrm{nm}$ of residual dispersion, providing performance benefits as compared to a fully compensated link without the regenerator. The theoretical analysis suggests that overlap of consecutive bits sets the limit to the chromatic dispersion tolerance.

\section{REFERENCES}

[1] A. H. Gnauck and P.J. Winzer, “Optical phase-shift-keyed transmission,” J. Lightwave Technol. 23 (1), 115-130 (2005).

[2] C. Xu, X. Liu, and X. Wei, “Differential phase-shift keying for high spectral efficiency optical transmissions,” IEEE J. Sel. Topics Quantum Electron. 10 (2), 281-293 (2004).

[3] C. Xu, X. Liu, L.F. Mollenauer, and X. Wei, "Comparison of return-to-zero differential phase-shift keying and ON-OFF keying in long-haul dispersion managed transmission,” IEEE Photon. Technol. Lett. 15, 617-619 (2003).

[4] H. Kim and A. Gnauck, "Experimental investigation of the performance limitation of DPSK systems due to nonlinear phase noise,” IEEE Photon. Technol. Lett.15, 320-322 (2003).

[5] K. Croussore, I. Kim, Ch. Kim, Y. Han, and G. Li, "Phase-and-amplitude regeneration of differential phase-shift keyed signals using a phase-sensitive amplifier,” Opt. Express 14, 2085-2094 (2006).

[6] D. Levandovsky, M. Vasilyev, and P. Kumar, “Amplitude squeezing of light by means of a phase-sensitive fiber parametric amplifier,” Opt. Lett. 24 (14), 984-986 (1999).

[7] K. Croussore and G. Li, "Phase Regeneration of NRZ-DPSK Signals Based on Symmetric-Pump Phase-Sensitive Amplification”, IEEE Photon. Tech. Lett. 19 (11) 864-866 (2007).

[8] R. Slavík et al, “All-optical phase and amplitude regenerator for next-generation telecommunications systems,” Nature Photonics 4 , 690-695 (2010).

[9] R.Slavík et al, "Coherent all-optical phase and amplitude regenerator of binary phase-encoded signals," to appear in IEEE J. Sel. Topic in Quantum Electron., DOI: 10.1109/JSTQE.2011.2136329.

[10] R.Slavík et al, “All-optical phase-regenerative multicasting of 40 Gbit/s DPSK signal in a degenerate phase sensitive amplifier,” European Conference on Optical Communications (ECOC) 2010, paper Mo1.A.2, Turin, Italy, Sept 2010.

[11] R. Weerasuriya et al., "Generation of frequency symmetric signals from a BPSK input for Phase Sensitive Amplification”, Optical Fiber Communication (OFC) 2010, paper OWT6, USA, March 2010.

[12] S. Sygletos et al., "Phase locking and carrier extraction scheme for phase sensitive amplification," $12^{\text {th }}$ Conference on Transparent optical networks (ICTON), paper Mo.C1.3, Munich, Germany, June 2010.

[13] R. Slavík et al, "Field-trial of an all-optical PSK regenerator ina 40Gbit/s 38 channel DWDM transmission experiment,” paper PDPA7, OFC 2011 Los Angeles 6-10 Mar 2011.

[14] C.-S. Brès, N. Alic, E. Myslivets and S. Radic, “1-to-40 Multicasting and Amplification of 40Gbps Channels in Wideband Parametric Amplifier,” PDP, OFC/NFOEC 2008.

[15] S. Kobayashi and T. Kimura, "Optical FM signal amplification by injection locked and resonant type semiconductor laser amplifiers," IEEE J. of Quantum Electron, vol. 18, no. 4, pp. 575-581, 1982.

[16] G. P. Agrawal, Nonlinear Fiber Optics. SanDiego, CA: Academic, 1995.

[17] J. Wang and K.Petermann, “Noise analysis of semiconductor lasers within the coherence collapse regime,” IEEE J. Quantum Electron.,vol. 21, no.1, pp. 39 (1991).

[18] K. Croussore and G. Li, "Phase and amplitude regeneration of differential phase-shift keyed signals using phase-sensitive amplification,” IEEE J. Sel. Topics Quantum. Electron., vol.14, no.3, pp. 648-658 (2008).

[19] M. Karlsson, H. Sunnerud, and B. E. Olsson, "PMD compensation using 2R and 3R regenerators (invited talk)," presented at the Eur. Conf. Opt. Commun. (ECOC), Stockholm, Sweden, 2004, We 1.4.2

[20] J. Kakande et. al, "Saturation Effects In Degenerate Phase Sensitive Fiber Optic Parametric Amplifiers," European Conference on Optical Communications (ECOC) 2010, paper Th.10.C.2, Turin, Italy, Sept 2010

[21] C. Lundström, B. Corcoran, Z. Tong, M. Karlsson and P. A. Andrekson, "Phase and amplitude transfer function of a saturated phase-sensitive parametric amplifier," accepted for European Conference on Optical Communications (ECOC) 2011, Geneva, Switzerland, Sept 2011.

[22] A. Bogris and D. Syvridis, "RZ-DPSK signal regeneration based on dual-pump phase-sensitive amplification in fibers,” IEEE Photon. Technol. Lett., vol. 18, no. 20, pp. 2144-2146 ( 2006).

[23] A. Fragkos, A. Bogris, D. Syvridis, R. Phelan, J. O'Carroll, B. Kelly, J. O'Gorman, “Amplitude Regeneration of Phase Encoded Signals Using Injection Locking in Semiconductor Lasers,” OFC/NFOEC 2011, Los Angeles, USA.

[24] R. Phelan, B.Kelly, J. O’Carroll, C. Herbert, A. Duke and J. O’Gorman, “- $40^{\circ} \mathrm{C}<\mathrm{T}<95^{\circ} \mathrm{C}$ mode-hop free operation of an uncooled AlGaInAs-MQW discrete-mode laser diode with emission at $\lambda=1.3 \mu \mathrm{m}$.” Electron. Lett. 45, 43-45 (2009).

[25] R.D.Li, P.Kumar, and W. L. Kath, “Dispersion compensation with phase sensitive optical amplifiers,” J. Lightwave Technol., vol. 12, no. 3, pp.541-549 (1994).

[26] L. Grüner-Nielsen et al., “A Silica Based Highly Nonlinear Fibre with Improved Threshold for Stimulated Brillouin Scattering,” paper Tu.4.D.3, ECOC 2010. 